\title{
Risk-Taking Behavior for HIV Acquisition during Pregnancy in Porto Alegre, Brazil
}

\author{
Nava Yeganeh, ${ }^{1,2}$ Ivana Varella, ${ }^{3}$ Breno Riegel Santos, ${ }^{3}$ Marineide Gonçalves de Melo, ${ }^{3}$ \\ Mariana Simon, ${ }^{3}$ Taui Melo, ${ }^{3}$ and Karin Nielsen-Saines ${ }^{1,2}$ \\ ${ }^{1}$ Department of Pediatrics, David Geffen School of Medicine at UCLA, Los Angeles, CA 90095, USA \\ ${ }^{2}$ UCLA Division of Infectious Disease-MDCC 22-442, 10833 LeConte Avenue, Los Angeles, CA 90095, USA \\ ${ }^{3}$ Servico de Infectologia, Hospital Nossa Senhora da Conceicao/GHC, Avenida Francisco Trein 596, 91350-200 Porto Alegre, RS, Brazil
}

Correspondence should be addressed to Nava Yeganeh, nyeganeh@mednet.ucla.edu

Received 31 July 2012; Revised 20 November 2012; Accepted 9 December 2012

Academic Editor: Consuelo Beck-Sagué

Copyright (c) 2012 Nava Yeganeh et al. This is an open access article distributed under the Creative Commons Attribution License, which permits unrestricted use, distribution, and reproduction in any medium, provided the original work is properly cited.

\begin{abstract}
Recent studies suggest that acquisition of HIV-1 infection during pregnancy and breastfeeding is associated with a high risk of HIV mother-to-child transmission. This study evaluates risk factors associated with HIV acquisition during pregnancy in women delivering at a large metropolitan medical facility located in the south of Brazil. From February to August 2009, our group conducted a cross-sectional study assessing women's risk for HIV acquisition by administering an oral survey to peripartum women. Of 2465 participants, $42 \%(n=1046)$ knew that partner had been tested for HIV. During pregnancy, 82\% $(n=2022)$ of participants never used condoms; yet $97 \%(n=2399)$ practiced vaginal sex. Multivariate logistic regression analysis showed that patients with more years of education, in a relationship for more than 1 year, and who knew their own HIV status were more likely to know their partners' HIV status $(P<0.05)$. Those who were in relationship for more than 1 year and were married/living together were more likely to be comfortable discussing HIV testing with partners $(P<0.05)$. In conclusion, women in Brazil are at risk of HIV-infection during pregnancy as they remain sexually active, often do not know their sexual partner's HIV status, and have minimal condom use.
\end{abstract}

\section{Introduction}

HIV is a preventable chronic, life-threatening illness affecting fetal and maternal health, increasing both morbidity and mortality in both infected and noninfected children worldwide [1-3]. Given the lack of an effective vaccine and the complexity of life-long treatment, prevention of maternal to child transmission (PMTCT) continues to be a crucial public health need globally. It has been well established that pregnant women have a significantly higher risk of HIV acquisition during gestation and while breastfeeding than their nonpregnant counterparts, due to behavioral factors, such as decreased condom use, and biological factors such as immunosuppression [4, 5]. Prior research in England, in African countries, in the United States, and by our own group in Brazil demonstrates that despite universal antenatal testing of women, HIV infected babies are born to women who had negative HIV tests during early prenatal care, implicating seroconversion during pregnancy and breastfeeding
[6-11]. Furthermore, studies conducted at our institution in Porto Alegre, Brazil, demonstrated that women known to seroconvert during pregnancy have a much higher HIV MTCT rate, than those with an unknown seroconversion time (transmission rate of $33 \%$ in the first group versus $8.2 \%$ in the latter) [6]. In fact, recent evidence indicates that infant HIV infection related to new maternal HIV-1 infection after antenatal HIV screening could account for up to $40 \%$ of all ongoing mother-to-child transmission in low and middle income countries $[9,12]$. The source of these new infections during pregnancy is likely the mother's sexual partner. Therefore, there is a renewed interest in expanding PMTCT efforts to include the mother's sexual partner.

With the goal of trying to expand PMTCT to the entire family unit including the male partners, we performed this initial study to determine the proportion of pregnant women who have knowledge of their sexual partner's HIV status results at the time of labor and delivery and their comfort 
in discussing HIV testing with their sexual partners during prenatal care and/or labor and delivery as well as to evaluate their risk factors for HIV acquisition. We decided to perform the study in Porto Alegre because the prevalence of HIV in pregnant women there rose from $0.5 \%$ in 1997 to $6.5 \%$ in 2002 [13]. At Hospital Conceiçao, a large tertiary county medical center providing care to a large sector of the low to middle income population of the metropolitan area, the HIV seroprevalence rates range from 3 to $5 \%$ in pregnant women receiving prenatal care, and 5 to $10 \%$ among pregnant women without prenatal care [14]. Prior to study initiation, we hypothesized that women continue to participate in high risk behavior during pregnancy and will agree to discuss HIV testing with partners in order to optimize their infant's health.

\section{Methods and Materials}

We performed a cross-sectional study in a population of pregnant women who self-reported themselves as either HIV negative or HIV untested and who gave birth to a live born infant at Conceiçao Hospital, a government-run county hospital in Porto Alegre, Brazil. Inclusion criteria included women who were in a steady heterosexual relationship for more than 3 months and older than 18 years of age. Of note, per hospital protocol, all women admitted for labor and delivery receive rapid HIV testing on admission. For our study, all HIV positive women were excluded. From February 2009 to August 2009, 3211 women who fulfilled the entry criteria and were hospitalized for labor and delivery were approached for participation in the study. After informed consent by the mother was obtained, interviewers administered a confidential scripted oral survey in Portuguese when participant was not in active labor (pre- and post-partum), questioning them on topics such as demographics (age, race, employment, salary, residence location, etc.), time in their current relationship, risk factors for HIV acquisition (condom use, sexual practices, and symptoms/signs of STDs), knowledge of partner's HIV status, and comfort in speaking about HIV testing with sexual partner. Descriptive statistics were provided for all data collected. Two-sample $t$-test and Pearson chi-square statistics were used to analyze continuous and categorical outcomes, respectively. A multivariate logistic regression analysis was performed to evaluate parameters associated with woman's knowledge of partner's status and comfort in discussing HIV testing with partner. Variables included in the model were age, race, marital status, employment, incomes, condom use, and knowledge of women's own HIV status. Missing data was discarded if it constituted less than $1 \%$ of total sample size. All computations were done using STATA 10 statistical software (Statacorp, College Station, Texas). IRB approval was obtained through both the UCLA Institutional Review Board and the Grupo Conceiçao IRB.

\section{Results}

Two thousand four hundred and sixty-five women participated in the study. The most common reasons given for not
TABle 1: Demographics $(n=2465)$.

\begin{tabular}{lc}
\hline Mean age (Years) & 27 (range 18-47) \\
Mean years of education (Years) & 9 (range 0-21) \\
$\begin{array}{l}\text { Mean monthly household income } \\
\text { (Brazilian Reis) }\end{array}$ & 1249 (range 0-20,000) \\
Race & \\
$\quad$ White & $1766(72 \%)$ \\
Black & $431(17 \%)$ \\
Mulatto/mixed & $243(10 \%)$ \\
First nation/native Indian & $25(1 \%)$ \\
Marital status & \\
Married/living together & $2270(92 \%)$ \\
Single parent & $194(8 \%)$ \\
Length of relationship* & $194(8 \%)$ \\
3 months to 1 year & $1193(48 \%)$ \\
$1-5$ yrs & $1077(44 \%)$ \\
$>5$ yrs
\end{tabular}

${ }^{*}$ Missing one response.

participating in the study included lack of available partner $(n=81)$, missed contact (patient discharged or unavailable prior to interview) $(n=601)$, or women already diagnosed as HIV positive $(n=39$.) The mean age of participating women was 27 years of age, with a range from 18 to 47 years. Approximately 92\% $(n=2270)$ of women were living with or married to their partners, $92 \%(n=2270)$ were in a stable relationship for at least 1 year and $72 \%(n=1766)$ selfidentified their race as White (Table 1).

Evaluating participant's HIV risk factors revealed that almost all women were sexually active during pregnancy including $97 \%(n=2399)$ of women who practiced vaginal sex, $29 \%(n=729)$ who practiced oral sex, and $15 \%(n=$ $363)$ who practiced anal sex (Table 2). Eighty-two percent $(n=2022)$ of women stated that they never used condoms during pregnancy. All had received HIV testing during labor and delivery (per hospital protocol), and $89 \%(n=2190)$ knew their own HIV status. Forty-six percent $(n=1131)$ knew their partner had undergone HIV testing and 42\% ( $n=1042)$ knew the actual HIV test results. None of them stated that they knew their partners were HIV positive. When asked whether they felt comfortable discussing HIV testing with their sexual partner, $96 \%(n=2366)$ confirmed that they would feel comfortable having this discussion with their partners, and $4 \%(n=99)$ said they would prefer not to discuss it.

Table 3 shows factors that were felt to be associated with women's knowledge of partner's HIV status as well as their comfort in discussing HIV with their partner. Multivariate logistic regression analysis showed that patients with more years of education, who were in a relationship for more than 1 year, and who already knew their own status during pregnancy were more likely to know their partners' HIV status $(P<0.05)$. Patients who were in a relationship for more than 1 year, and married/living together were more 
TABLe 2: HIV Risk Factors $(n=2465)$.

\begin{tabular}{lc}
\hline Age at loss of virginity (mean year) & 16 (range $7-32)$ \\
Condom use during pregnancy & \\
Every time & $136(6 \%)$ \\
Most of the time $(>50 \%)$ & $66(3 \%)$ \\
Sometimes $(10-50 \%)$ & $133(5 \%)$ \\
Rarely $(<10 \%)$ & $97(4 \%)$ \\
Never & $2022(82 \%)$ \\
Denied being sexually active during pregnancy & $11(0.4 \%)$ \\
Sexual practices during pregnancy & \\
Vaginal & $2399(97 \%)$ \\
Oral & $729(29 \%)$ \\
Anal & $363(15 \%)$ \\
Knowledge of partner's status & $1046(42 \%)$ \\
Yes & $1419(58 \%)$ \\
No & \\
Comfortable discussing HIV with partner & $2366(96 \%)$ \\
Yes & $99(4 \%)$ \\
No
\end{tabular}

likely to be comfortable discussing HIV testing with their partners $(P<0.05)$.

Table 4 displays reasons why the 99 women did not feel comfortable discussing HIV testing with their partners. The most common reason was embarrassment $(23 \%, n=23)$, and stigma $(7 \%, n=7)$. Even after being prompted with responses, only $3 \%(n=3)$ stated they feared abandonment if HIV testing was discussed, 0 individuals indicated fear of physical abuse, and $1 \%(n=1)$ expressed concern for emotional abuse. Of the responses that were not prompted by interviewer ("Other" category), $22 \%(n=22)$ of women stated that they did not feel comfortable asking their partners to get HIV tested because their partner would refuse, $17 \%$ $(n=17)$ of women stated that it would be inconvenient for their partners to be tested because of other responsibilities, and $13 \%(n=13)$ started that they could not contact their partners either because they had left town or because they were in prison.

\section{Discussion}

Pregnant women in Porto Alegre are at risk of HIV acquisition since almost all remain sexually active, yet few consistently use condoms. The results are concerning in that it is likely that a large portion of cases of HIV PMTCT at the Conceiçao Hospital, the largest public tertiary institution in the metropolitan area of Porto Alegre, may be occurring because of maternal seroconversion during pregnancy. Of the last nine infants who acquired HIV from infected mothers between 2004 and 2006, 3 infants acquired it from women who are known to have seroconverted during pregnancy with a negative test in the first trimester and a positive HIV test during labor and delivery, 5 infants acquired HIV from mothers who had an unknown time of seroconversion, and 1 acquired it from a known HIV positive mother [6]. The risk is considerable particularly because the south of Brazil is the area of highest HIV seroprevalence (3-5\%) in the country $[6,15]$. More than half of the women in our study population did not know their partner's HIV status, placing them at risk for seroconversion during pregnancy. Fortunately, 96\% of women felt comfortable discussing HIV testing with their partners, which suggests it is feasible for women to agree to bring in their partners for HIV testing during prenatal care. Of the $4 \%$ who did not feel comfortable discussing HIV testing with their partners, the most common reasons included embarrassment and concern that their partner would refuse testing, both of which could be addressed through appropriate counseling. Almost none of participants stated they had genuine fears of physical or emotional abuse, even when verbally prompted for these responses. Partner violence against HIV positive women has been observed in $4 \%$ to $6.6 \%$ of women who disclose their HIV status to their partners $[16,17]$. Specifically in the state of Rio Grande do Sul, where this institution is located, a study conducted in 2009 showed that $43 \%$ of women in the general population had experienced lifetime violence and $18.2 \%$ reported violence during pregnancy [18]. Although women in our study did not indicate any concerns regarding partner violence, multidisciplinary involvement and counseling efforts should be prioritized so that women are protected from violence if the approach of partner testing is implemented during pregnancy or in the peripartum period.

Our data demonstrated that being in a relationship for longer than one year was associated with higher rates of knowledge of one's partner HIV status and increased degree of comfort in discussing HIV testing, suggesting that women feel more secure discussing more sensitive issues, such as sexually transmitted infections, if the relationship has proven stable by withstanding the test of time. Women who felt more comfortable discussing HIV with their partner were also significantly more likely to be married/living with their partners, which may reflect a similar sense of intimacy. However, it is important to note that in a study done in Kenya, women who were married were more likely to seroconvert during pregnancy and the postpartum period, so married couples may represent a high risk group for seroconversion [9].

Interestingly, our analysis also showed that women who knew their own HIV status were also more likely to know their partner's HIV status. Likely, these women had received education regarding HIV as part of the voluntary counseling and testing and had greater awareness of the risks of acquiring HIV from their partners as a sexually transmitted disease. Thus, the experience of receiving HIV voluntary counseling and testing (VCT) may have motivated them to discuss HIV testing with their sexual partners.

Prior studies in Brazil and in other countries have shown that decreased education is associated with lower rates of HIV VCT in pregnant and nonpregnant women [19-22]. Our study shows that education is not only associated with women receiving appropriate VCT for themselves, but also with knowing the HIV status of their partner. Therefore, education may play a significant role both in pregnant 
TABLE 3: Factors associated with participant's partners HIV status and endorsing comfort in speaking to partner about HIV testing**.

\begin{tabular}{|c|c|c|c|c|c|c|}
\hline & \multicolumn{3}{|c|}{ Knowledge of partner's HIV status? } & \multicolumn{3}{|c|}{ Comfortable speaking to partner about HIV? } \\
\hline & $\begin{array}{c}\text { Yes } \\
1046(42 \%)\end{array}$ & $\begin{array}{c}\text { No } \\
1419(58 \%)\end{array}$ & $P$ value* & $\begin{array}{c}\text { Yes } \\
2366(96 \%)\end{array}$ & $\begin{array}{c}\text { No } \\
99(4 \%)\end{array}$ & $P$ value* \\
\hline Age & $26.6( \pm 6.2)$ & $26.7( \pm 6.3)$ & 0.55 & $26.7( \pm 6.3)$ & $27( \pm 6.2)$ & 0.22 \\
\hline Race & & & 0.94 & & & 0.31 \\
\hline White & $767(73 \%)$ & $997(70 \%)$ & & $1702(72 \%)$ & $64(64 \%)$ & \\
\hline Black/AA & $168(16 \%)$ & $263(19 \%)$ & & $412(17 \%)$ & $19(19 \%)$ & \\
\hline Mulatto/Pardo & $101(10 \%)$ & $143(10 \%)$ & & $229(10 \%)$ & $14(14 \%)$ & \\
\hline Native Indian & $9(1 \%)$ & $16(1 \%)$ & & $23(1 \%)$ & $2(2 \%)$ & \\
\hline Marital status & & & 0.577 & & & $<0.001$ \\
\hline Married/living together & $978(94 \%)$ & $1319(93 \%)$ & & $2220(94 \%)$ & $79(80 \%)$ & \\
\hline Single/divorced & $67(6 \%)$ & $99(7 \%)$ & & $146(6 \%)$ & $20(20 \%)$ & \\
\hline Time in relationship & & & 0.024 & & & 0.021 \\
\hline 3 months to 1 year & $69(7 \%)$ & $125(9 \%)$ & & $177(7 \%)$ & $17(17 \%)$ & \\
\hline$>1$ year & $977(93 \%)$ & $1295(91 \%)$ & & $2188(93 \%)$ & $82(83 \%)$ & \\
\hline Employment & & & 0.59 & & & 0.28 \\
\hline Yes & $389(38 \%)$ & $549(39 \%)$ & & $911(39 \%)$ & $28(29 \%)$ & \\
\hline No & $641(62 \%)$ & $864(61 \%)$ & & $1436(61 \%)$ & $70(71 \%)$ & \\
\hline Education (years) & $9.3( \pm 3)$ & $8.7( \pm 3)$ & $<0.005$ & $9( \pm 3)$ & $9( \pm 3.5)$ & 0.9 \\
\hline Income (Brazilian Reais) & $1327( \pm 1083)$ & $1190( \pm 955)$ & 0.1 & $1257( \pm 1020)$ & $1046( \pm 824)$ & 0.1 \\
\hline Knowledge of own status & & & $<0.000$ & & & 1 \\
\hline Yes & $960(92 \%)$ & $1228(87 \%)$ & & $2102(89 \%)$ & $88(89 \%)$ & \\
\hline No & $83(8 \%)$ & $191(13 \%)$ & & $263(11 \%)$ & $11(11 \%)$ & \\
\hline Condom use (pregnancy) & & & 0.18 & & & 0.47 \\
\hline Yes & $197(19 \%)$ & $235(17 \%)$ & & $416(18 \%)$ & $16(17 \%)$ & \\
\hline No & $843(81 \%)$ & $1177(83 \%)$ & & $1943(82 \%)$ & $79(83 \%)$ & \\
\hline
\end{tabular}

* Adjusted for age, race, marital status, employment, incomes, condom use, and knowledge of women's own HIV status.

** Missing responses discarded if less than $1 \%$ of total number of participants.

TABLE 4: Reasons women feel uncomfortable discussing HIV testing with partners.

\begin{tabular}{lc}
\hline & $\begin{array}{c}\text { Number of } \\
\text { affirmative responses } \\
(n=99)\end{array}$ \\
\hline Ashamed & $23(23 \%)$ \\
Stigma & $7(7 \%)$ \\
Not worried about infection & $5(5 \%)$ \\
Abandon & $3(3 \%)$ \\
Anxiety & $2(2 \%)$ \\
Emotional abuse & $1(1 \%)$ \\
Physical abuse & 0 \\
Other responses listed below & $62(62 \%)$ \\
$\quad$ Inconvenient (traveling/working) & $17(17 \%)$ \\
Spouse not available/in prison & $13(13 \%)$ \\
Fear of needles/hospital & $2(2 \%)$ \\
Spouse does not want to/refuses & $22(22 \%)$ \\
$\quad$ No response & $8(8 \%)$ \\
\hline
\end{tabular}

women receiving appropriate care for themselves, and also in any efforts involving HIV VCT of their partners.

The limitations of our study include the fact that data was collected through self-reports by an interviewer previously unknown to the patient. As the survey included very personal information such as condom use and sexual practices/risk factors it is possible that patients did not disclose intimate information as they were inhibited by the presence of the interviewer. Our surveys were conducted in a low-income county hospital in the south of Brazil, which may also limit extrapolation of our data to other settings. However, given that the patient population was comprised of women referred to Conceiçao through a vast network of peripheral clinics throughout the city, our survey likely captured a representative sample of the general population of low to middle income women of Porto Alegre and adjacent neighborhoods. We focused on women who had a sexual partner for greater than 3 months, which may not be generalizable to the entire population of pregnant women. However, we assumed people who were not with a steady heterosexual partner for greater than 3 months may actually be at higher risk of HIV acquisition since having multiple partners increases risk of HIV transmission [23].

There may be a real need to screen partners of women undergoing VCT for prenatal care, as a public health measure to effectively reduce sexual transmission and MTCT of HIV-1, particularly in areas of high HIV-1 seroprevalence. Data demonstrates that women not only have a higher rate of infection during pregnancy, but also have higher mother-to-child transmission rates during pregnancy and 
breastfeeding [4-6, 9]. Studies in Kenya, Swaziland, South Africa, and Botswana have shown HIV seroincidence rates in pregnant women ranging from 1.3 to 16.8 infections/100 person years $[9,10,24,25]$. These high rates are not only concerning when considering their implications for women's health, but also doubly alarming since transmission rates to the infant are higher during acute maternal HIV infection $[6,8,11]$. Our study highlights the importance of protecting women during this vulnerable time, since most remain sexually active without condom use. Hopefully, these results can inform future efforts to expand prenatal care to involve not only the mother, but the entire family as well. In the future, interventions can focus on testing partners of pregnant women during prenatal care, and if the partners refuse testing, potentially offering appropriate antiretroviral prophylaxis to women in very high risk settings during this susceptible period.

\section{Conflict of Interests}

The participants of this study deny any conflict of interests.

\section{Acknowledgments}

This work was supported by the following grants: NIH UO1 AI/69401-01, the UCLA Program in Global Health/UCLA AIDS Institute's seed grant program: International Prevention and Policy Research for HIV/AIDS (2008), UCLA AIDS Institute's Fellowship Grant (2009), and the UCLA Pediatric Translational Research Program Seed Grant (2010). The data was presented in the Pediatric Academic Society conference in May 2010 in Vancouver, Canada.

\section{References}

[1] A. Slogrove, B. Reikie, S. Naidoo et al., "HIV-exposed uninfected infants are at increased risk for severe infections in the first year of life," Journal of Tropical Pediatrics, vol. 58, no. 6, pp. 505-508, 2012.

[2] UNAIDS, Joint United Nations Programme on HIV/Aids, 2008 Report on the Global Aids Epidemic, vol. 216, UNAIDS, Geneva, Switzerland, 2008.

[3] W. Sophie, G. Calder, and T. Ahimbisibwe, Taking Better Care? Review of a Decade of Work with Orphans and Vulnerable Children in Rakai, Uganda, Save the Children, 2004.

[4] R. H. Gray, X. Li, G. Kigozi et al., "Increased risk of incident HIV during pregnancy in Rakai, Uganda: a prospective study," The Lancet, vol. 366, no. 9492, pp. 1182-1188, 2005.

[5] N. R. Mugo, R. Heffron, D. Donnell et al., "Increased risk of HIV-1 transmission in pregnancy: a prospective study among African HIV-1-serodiscordant couples," AIDS, vol. 25, no. 15, pp. 1887-1895, 2011.

[6] K. Nielsen-Saines, M. Melo, I. Varella et al., "Primary HIV1 infection during pregnancy: high rate of HIV-1 Mtct in a cohort of patients in Southern Brazil," Retrovirology, vol. 5, supplement 1, article 01, 2008.

[7] S. S. Struik, G. Tudor-Williams, G. P. Taylor et al., "Infant HIV infection despite "universal" antenatal testing," Archives of Disease in Childhood, vol. 93, no. 1, pp. 59-61, 2008.
[8] D. Moodley, T. Esterhuizen, L. Reddy et al., "Incident HIV infection in pregnant and lactating women and its effect on mother-to-child transmission in South Africa," Journal of Infectious Diseases, vol. 203, no. 9, pp. 1231-1234, 2011.

[9] L. Lu, K. Legwaila, C. Motswere, M. Smit, W. Jimbo, and T. Creek, "HIV incidence in pregnancy and the first postpartum year and implications for Pmtct programs; francistown, Botswana," in Proceedings of the 16th Conference on Retroviruses and Opportunistic Infections, 2009, Abstract no. 91.

[10] J. Kinuthia, J. N. Kiarie, C. Farquhar et al., "Cofactors for HIV-1 incidence during pregnancy and postpartum period," Current HIV Research, vol. 8, no. 7, pp. 510-514, 2010.

[11] S. Singh, "HIV seroconversion during pregnancy and motherto-child HIV transmission: data from the Enhanced Perinatal Surveillance Project, United States, 2005-2010," in Proceedings of the International AIDS Conference, Washington, DC, USA, 2012.

[12] L. F. Johnson, K. Stinson, M. L. Newell et al., "The contribution of maternal HIV seroconversion during late pregnancy and breastfeeding to mother-to-child transmission of HIV," Journal of Acquired Immune Deficiency Syndromes, vol. 59, no. 4, pp. 417-425, 2012.

[13] V. G. Veloso, F. I. Bastos, M. C. Portela et al., "HIV rapid testing as a key strategy for prevention of mother-to-child transmission in Brazil," Revista de Saude Publica, vol. 44, no. 5, pp. 803-811, 2010.

[14] M. Melo, I. Varella, A. Castro et al., "HIV rapid testing of previously untested sexual partners of HIV-negative women delivering at a large public hospital- a case finding strategy," in Proceedings of the 16th Conference on Retroviruses and Opportunistic Infections, 2010, Abstract no. 1003.

[15] UNAIDS, ASMS, and PMPA, Aids Em Porto Alegre, Rs, Equipe de Vigilancia das Doencas Transmissiveis, 2011.

[16] A. C. Gielen, K. A. McDonnell, J. G. Burke, and P. O'Campo, "Women's lives after an HIV-positive diagnosis: disclosure and violence," Maternal and Child Health Journal, vol. 4, no. 2, pp. 111-120, 2000.

[17] P. Gaillard, R. Melis, F. Mwanyumba et al., "Vulnerability of women in an African setting: lessons for mother-to-child HIV transmission prevention programmes," AIDS, vol. 16, no. 6, pp. 937-939, 2002.

[18] P. Manzolli, M. A. A. Nunes, M. I. Schmidt et al., "Violence and depressive symptoms during pregnancy: a primary care study in Brazil," Social Psychiatry and Psychiatric Epidemiology, vol. 45, no. 10, pp. 983-988, 2010.

[19] M. Fabiani, A. Cawthorne, B. Nattabi, E. O. Ayella, M. Ogwang, and S. Declich, "Investigating factors associated with uptake of HIV voluntary counselling and testing among pregnant women living in North Uganda," AIDS Care, vol. 19, no. 6, pp. 733-739, 2007.

[20] P. L. Hutchinson and X. Mahlalela, "Utilization of voluntary counseling and testing services in the Eastern Cape, South Africa," AIDS Care, vol. 18, no. 5, pp. 446-455, 2006.

[21] K. Peltzer, G. Matseke, T. Mzolo, and M. Majaja, "Determinants of knowledge of HIV status in South Africa: results from a population-based HIV survey," BMC Public Health, vol. 9, article 174, 2009.

[22] H. Rosa, M. Z. Goldani, T. Scanlon et al., "Barriers for HIV testing during pregnancy in Southern Brazil," Revista de Saude Publica, vol. 40, no. 2, pp. 220-225, 2006.

[23] Q. A. Karim, S. Sibeko, and C. Baxter, "Preventing HIV infection in women: a global health imperative," Clinical Infectious Diseases, vol. 50, supplement 3, pp. S122-S129, 2010. 
[24] S. Mepham, R. Bland, J. Ndirangu, and M. L. Newell, "HIV incidence and associated socio-economic factors in a prospective cohort of antenatal and postpartum women in rural, Northern Kwazulu-Natal," in Proceedings of the 5th IAS Conference on HIV Pathogenesis, Treatment and Prevention, 2009.

[25] M. P. Kieffer, B. Nhlabatsi, M. Mahdi, H. J. Hoffman, K. Kudiabor, and C. M. Wilfert, "Improved detection of incident HIV infection and uptake of PMTCT services in labor and delivery in a high HIV prevalence setting," Journal of Acquired Immune Deficiency Syndromes, vol. 57, no. 4, pp. e85-e91, 2011. 


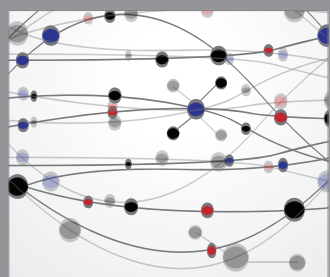

The Scientific World Journal
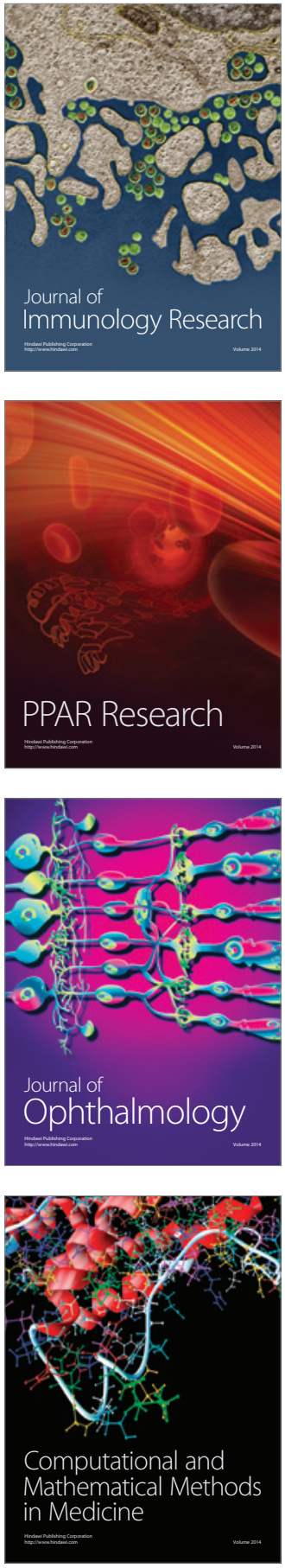

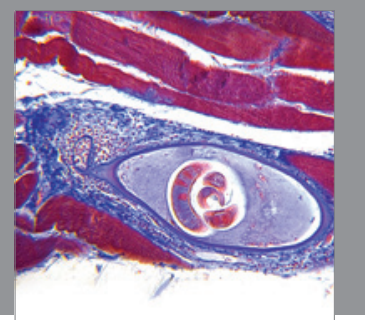

Gastroenterology

Research and Practice
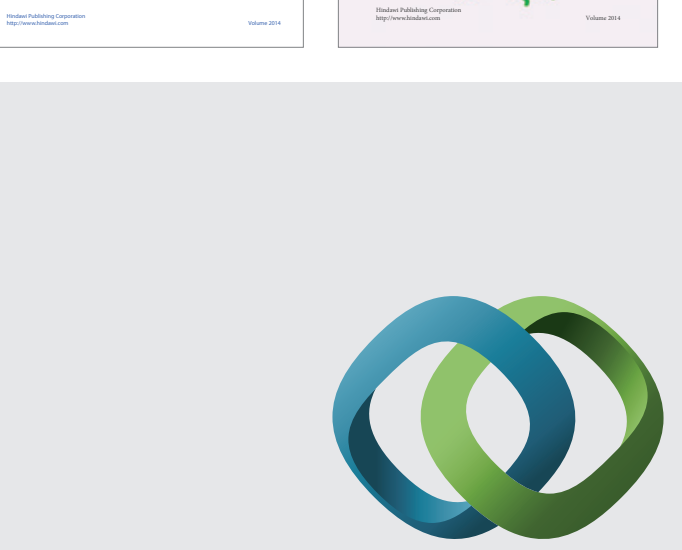

\section{Hindawi}

Submit your manuscripts at

http://www.hindawi.com
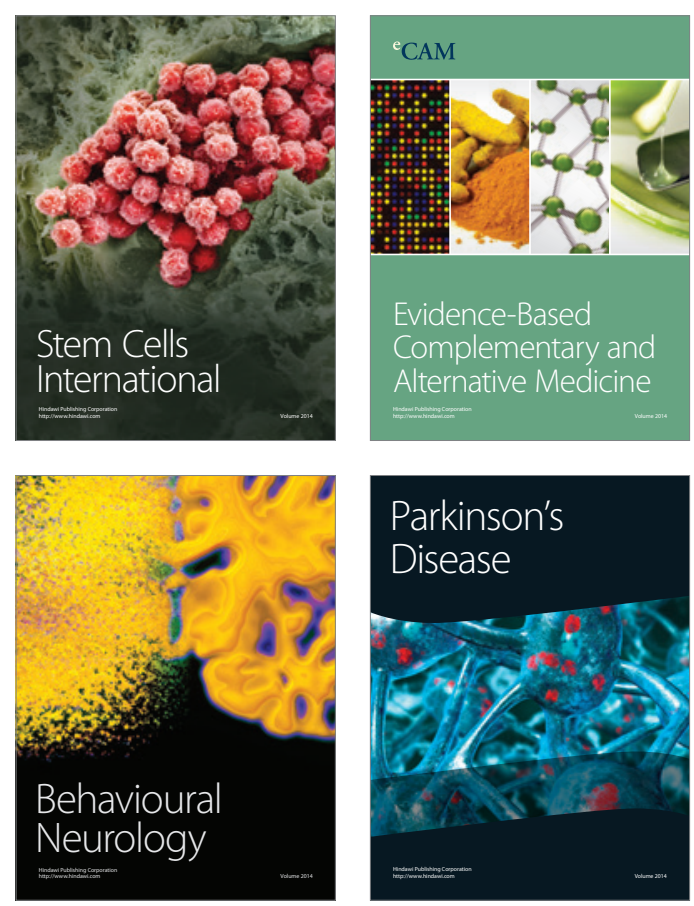

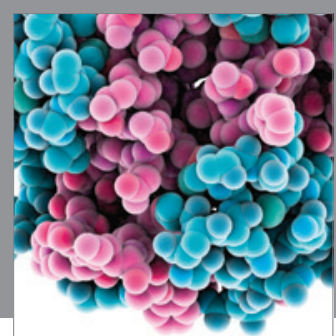

Journal of
Diabetes Research

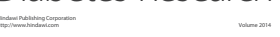

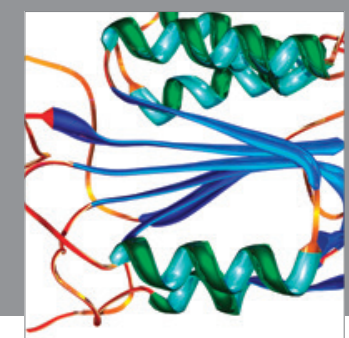

Disease Markers
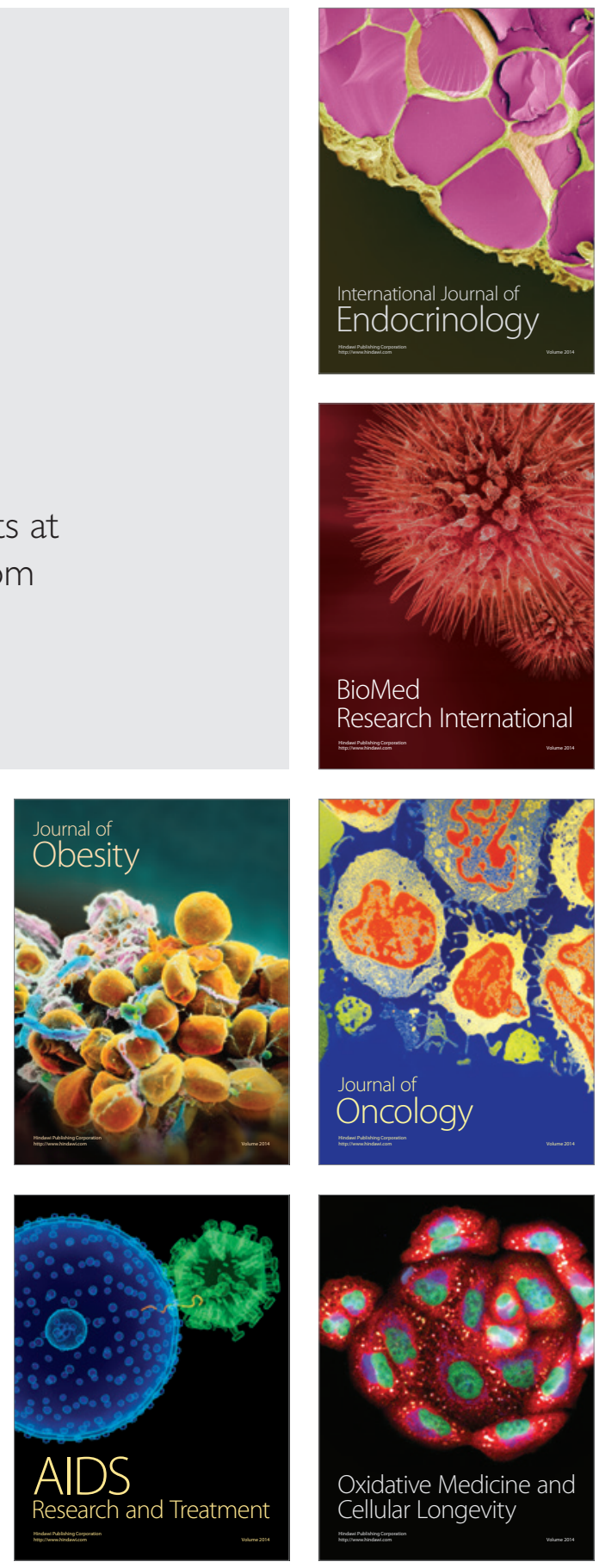